\title{
Kortikosteroide haben bei COPD fast ausgedient
}

\author{
Bei Patienten mit mittelgradiger und schwerer COPD verhindert die duale Bronchodilatation \\ Exazerbationen besser als eine steroidhaltige Therapie. Es ist ein Umdenken angesagt.
}

_ In der Studie FLAME ging es um die Medikation von Patienten mit mittelgradiger oder schwerer COPD. Ihre Einsekundenkapazität $\left(\mathrm{FEV}_{1}\right)$ lag zwischen $25 \%$ und $60 \%$ des Sollwerts. Über 52 Wochen nahmen 1.680 Patienten einmal täglich eine Fixkombination aus dem lang wirksamen Betaagonisten (LABA) Indacaterol $(110 \mu \mathrm{g})$ und dem lang wirksamen Anticholinergikum (LAMA) Glycopyrronium $(50 \mu \mathrm{g})$ ein. 1.682 Patienten nahmen zweimal täglich eine Fixkombination aus dem LABA Salmeterol $(50 \mu \mathrm{g})$ und dem inhalativen Steriod (ICS) Fluticason $(500 \mu \mathrm{g})$ ein.

Unter Indacaterol/Glycopyrronium reduzierte sich die jährliche Exazerbationsrate auf 3,59 gegenüber 4,03 unter Salmeterol/Fluticason. Der Unterschied von $11 \%$ war statistisch signifikant ( $\mathrm{p}=$ 0,003). Auch verging mehr Zeit bis zur ersten Exazerbation (71 vs. 51 Tage, $\mathrm{p}<$ 0,001). Die jährliche Rate moderater oder schwerer Exazerbationen lag unter der LABA/LAMA-Kombination ebenfalls niedriger ( 0,98 vs. $1,19, \mathrm{p}<0,001)$. Gleiches galt für die Zeit bis zur ersten moderaten oder schweren Exazerbation (Hazard Ratio 0,78, p < 0,001) bzw. zur ersten schweren Exazerbation (Hazard Ratio 0,81, p = 0,046).

Die Effekte waren davon unabhängig, ob die Patienten zu Studienbeginn eine Eosinophilie von weniger oder mehr als $2 \%$ hatten. Unerwünschte Ereignisse und Todesfälle traten in beiden Gruppen gleich häufig auf. Die Pneumonierate war unter Indacaterol/Glycopyrronium geringer $(3,2 \%$ vs. $4,8 \%, \mathrm{p}=0,02)$.

- Wedzicha JA et al. (FLAME Investigators). IndacaterolGlycopyrronium versus Salmeterol-Fluticasone for COPD. N Engl J Med. 2016;374:2222-34

\section{KOMMENTAR}

ICS sollen bei Patienten mit schwerer oder sehr schwerer COPD eingesetzt werden, die unter wiederholten Exazerbationen und trotz lang wirksamer Bronchodilatatoren unter Symptomen leiden. Das sind eigentlich weniger als 2\% der COPD-Patienten - doch ICS werden viel häufiger gegeben. Es ist ein Umdenken erforderlich. Besteht bei einem $\mathrm{Pa}$ tienten eine reine COPD - ohne Hinweis auf ein gleichzeitig bestehendes Asthma-, rücken ICS deutlich in den Hintergrund. Sowieso erhöhen alle ICS das Pneumonierisiko, auch wenn bei korrekter Indikationsstellung der Nutzen überwiegt. Es kommt also auf eine sorgfältige Identifikation der wirklich geeigneten Patienten an. Neben Erkrankungsschwere und Exazerbationsrate wird dabei nun auch die Frage wichtig, ob bereits eine duale Bronchodialation erfolgt.

Dr. med. J. de Zeeuw

\section{Dieses Geschwür ist alles andere als banal}
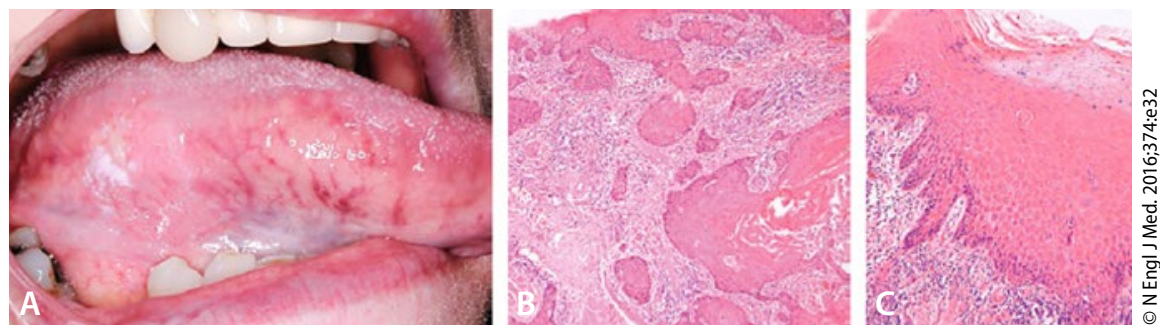

A: Ulkus am Zungenrand. B: Histologische Darstellung des Plattenepithelkarzinoms (HE-Färbung). C: Leichte Dysplasie in der fleckig geröteten Umgebung.

Eine 73-jährige Frau war über einen weißen Fleck und ein kleines Geschwür am rechten Zungenrand, die seit etwa einem Jahr bestanden, beunruhigt. Beim Essen empfand sie leichte Schmerzen an der Stelle. Sie war Nichtraucherin und trank keinen Alkohol. Bei der körperlichen Untersuchung waren keine vergrößerten Lymphknoten feststellbar. Am rechten Zungenrand fand sich ein $7 \times 3 \mathrm{~mm}$ großes Ulkus mit glattem Grund, induriertem Rand und fleckig geröteter Umgebung (Abb. A). Die Biopsie ergab ein invasives Plattenepithelkarzinom (Abb. B). Eine Biopsie aus der Stelle mit fleckiger Rötung zeigte hyperchromatische und polymorphe Zellen der Basalmembran. Dieser Befund entsprach einer leichten Dysplasie (Abb. C). In der CT ergaben sich keine Hinweise für eine Beteiligung von Halslymphknoten. Nach einer Ex- zision weit im Gesunden wies die Patientin bei einer Nachuntersuchung ein Jahr später keine Hinweise für ein Rezidiv auf.

Die Fünf-Jahres-Überlebensrate von Patienten mit Plattenepithelkarzinomen der Zunge hat sich in den letzten Jahren trotz des medizinischen Fortschritts kaum verändert und liegt bei deprimierenden 50\%. Dies liegt wahrscheinlich an der späten Diagnosestellung, da Ulzerationen und Verhärtungen im Bereich der Zunge von den Patienten lange bagatellisiert werden und keinen Anlass zu einem Arztbesuch geben. Der vorliegende Fall belegt den Wert mehrerer Biopsien an verschiedenen, unterschiedlich aussehenden Stellen, da die Ausdehnung des Karzinoms makroskopisch nur schwer festzustellen ist.

Prof. Dr. med. H. S. FüeßI

- Albuquerque RPP, Richards A

(r.p.p.d.albuquerque@bham.ac.uk).Squamous-cell carcinoma of the tongue. N Engl J Med. 2016;374:e32 